\title{
Développement d'un concept et d'un processus de gestion de l'identité numérique d'un produit en établissement de santé
}

\author{
Jean-François Bussières, Denis Lebel, Serge Voytenko et Guillaume Vaquer
}

\section{INTRODUCTION}

A u Canada, il existe plus de 22000 médicaments et plus de 44000 instruments médicaux' sous l'égide de la Loi sur les aliments et drogues et de sa réglementation. Avec l'entrée en vigueur du Règlement sur les produits de santé naturels en 2006, on estime qu'il existe en plus des médicaments, de 40000 à 50000 produits de santé naturels sur le marché canadien, qui feront potentiellement l'objet d'une évaluation par Santé Canada au cours des prochaines années². Les professionnels de la santé et les patients doivent composer avec un nombre très élevé et croissant de produits, d'étiquetages et de formats. Le cadre législatif prévoit les modalités entourant l'étiquetage externe pour la vente et l'étiquetage interne pour l'utilisation des médicaments et produits de santé naturels. Toutefois, il n'existe aucune obligation pour le fabricant de rendre disponible aux professionnels de la santé une version électronique standardisée des illustrations des différentes formes d'emballage de ces produits. En dépit de cette réglementation concernant l'étiquetage, certains fabricants commercialisent déjà au Canada des plaquettes alvéolées, dont l'étiquetage n'est pas jugé sécuritaire en établissement de santé3.

En vertu des lignes directrices américaines et canadiennes, la prestation sécuritaire de soins repose sur une distribution unitaire des médicaments et des produits de santé naturels en établissement de santét. Cette distribution unitaire repose sur une capacité d'identifier adéquatement chaque dose de médicament. La vision 2015 de la Société canadienne des pharmaciens d'hôpitaux prévoit que $75 \%$ des départements de pharmacie devront offrir une distribution unitaire à plus de 90 $\%$ des lits de leur établissement ${ }^{5}$. L'enquête canadienne sur la pharmacie hospitalière de 2007-2008 révèle que $66 \%$ des personnes ayant répondu à l'enquête offre une telle distribution pour une proportion moyenne de $81 \%$ des lits de courte durée 6 . On reconnaît qu'il existe une cinquantaine d'étapes au circuit du médicament en établissements de santé, de l'acquisition du médicament, en passant par la prescription, la validation et la distribution ainsi que l'administration au patient ${ }^{7}$. Chacune de ces étapes comporte de nombreux risques d'erreur médicamenteuse $e^{8}$.

L'Institute for Safe Medication Practices (ISMP), en EtatsUnis, reconnait 10 éléments clés d'un circuit du médicament sécuritaire, soit l'information sur le patient, l'information sur le médicament, la communication entre professionnels, l'étiquetage et l'emballage, l'entreposage et la standardisation des stocks, l'acquisition, l'utilisation et l'entretien des équipements, l'environnement, la compétence et la formation du personnel, l'instruction donnée au patient, la gestion de la qualité et des risques?. Afin de réduire les risques, on a retenu plusieurs technologies pour assurer une utilisation plus sécuritaire des médicaments (p. ex. prescripteurs électroniques, lecteurs de codes-barres à la pharmacie et au chevet du patient, pompes intelligentes, sélection des médicaments requis à partir de contenants marqués de puces activées par radio-fréquence). La mise en place des éléments clés proposés par ISMP et l'implantation de ces technologies peuvent avoir potentiellement un effet favorable sur la réduction des risques. Toutefois, plusieurs éléments clés démontrent que la disponibilité de l'information tout au long du processus et les communications des professionnels entre eux et des professionnels avec les patients sont très importantes. Créer et gérer une nouvelle base de données comportant des photos, basée sur l'identité numérique des médicaments, peut permettre de disposer d'un outil important. Cet élément est à la base de notre proposition visant à améliorer la qualité du circuit du médicament. La structure de cette nouvelle base de données est dictée par notre concept. Cette nouvelle banque complète efficacement les bases de données patients et médicaments déjà en notre possession et utilisées quotidiennement. À ce jour, l'Association des 
pharmaciens du Canada (APC) produit et détient une banque de données d'environ 1500 images couleur de médicaments commercialisés au Canada, principalement sous formes orales solides. Les images sont en format JPG $(300 \times 300$ pixels $)$ et sont archivées dans une banque de données contenant le numéro d'identification du médicament (DIN), le nom générique, le nom commercial, la teneur, la forme, la couleur, le contenu des inscriptions sur la forme et le fabricant. Cette banque de données est publiée annuellement en version papier et électronique du Compendium des produits et spécialités. L'APC envisage de rendre disponible cette banque de données à certains utilisateurs, notamment aux établissements de santé. Il semble très clair qu'un groupe canadien doit prendre le leadership afin d'assurer le développement d'une banque d'images complète à l'échelle canadienne.

À l'heure actuelle, très peu de données ont été publiées sur l'utilisation d'images de médicaments en pharmacie. Katsuma et coll. ont évalué l'efficacité de l'ajout d'une image imprimée sur le sac de médicaments pour la prévention d'erreurs de distribution et noté un impact favorable sur la détection d'erreurs de distribution ${ }^{10}$. Fox et coll. rapportent une utilisation d'images dans un cadre pédagogique pour la formation de professionnels ${ }^{11}$.

Lobjectif de cet article est de décrire le développement d'un concept et d'un processus de gestion de l'identité numérique d'un produit en établissement de santé.

\section{IDENTITÉ NUMÉRIQUE}

L'identité numérique d'un produit est définie comme étant toute information liée à celui-ci et enregistrée sous forme numérique. Cela concerne principalement les médicaments, mais le concept peut aussi être appliqué à d'autres produits et fournitures.

Cette identité peut comporter différents numéros d'identification (p. ex. numéro DIN délivré par Santé Canada, son numéro GTIN (Global Trade Identification Number), le numéro de produit chez le fabricant et le distributeur, le numéro de produit chez le groupe d'achats, le numéro de produit au sein de l'établissement), des documents numériques (p. ex. monographie du médicament, publications pertinentes, correspondance avec le fabricant), des images du produit dans son contenant original ou hors de son contenant (p. ex. format d'achat, comme la caisse de 12 , format d'utilisation au département de pharmacie, comme le pot de 100 comprimés, format d'utilisation au chevet du patient, comme le comprimé ensaché), avec ou sans modifications effectuées par le pharmacien et son équipe (p. ex. ensachage, préparation en mini-sacs ou seringues) et des fichiers sonores de la prononciation des noms du médicament (p. ex. prononciation du nom générique, du nom commercial). Actuellement, une grande partie de cette information existe de façon plus ou moins complète dans des banques de données expertes (p. ex.
Micromedex de Thomson Healthcare, Compendium des produits et spécialités de l'APC), des banques de données transactionnelles (p. ex. Pharmaclick de Mckesson Canada), des banques de données informatives (p. ex. Commerce Santé du groupe d'achats Approvisionnements Montréal), des dossiers pharmacologiques informatisés (p. ex. GesPharx de CGSI Solutions TI Inc.). Toutes ces banques ont en commun les éléments suivants : une banque de données, une fiche produit comportant un numéro d'identification par médicament et plusieurs variables associées ; toutefois, la plupart de ces banques sont difficiles à interfacer compte tenu qu'il n'existe pas de numéro d'identification unique à l'échelle du format d'utilisation et d'administration du médicament en établissement de santé ${ }^{12}$. Certains logiciels prévoient déjà l'utilisation d'images pour rendre le circuit du médicament plus sécuritaire (p. ex. PacmedServer pour l'ensacheuse Pacmed de Mckesson). Toutefois, ce système serait optimal s'il reposait sur une banque de données complète, dont les informations jugées pertinentes seraient définies sur le plan national.

Pour que la gestion de l'identité numérique soit applicable en établissement de santé, il est impératif d'établir un numéro unique destiné au format de distribution et d'utilisation. Le DIN émis par Santé Canada ne peut être utilisé, parce qu'il s'applique à un ingrédient actif et à une teneur, sans prise en compte du format. Dans le circuit du médicament, le numéro d'identification requis doit tenir compte du format d'emballage (p. ex. pot de 100, pot de 500, pot de 1000). Dans la perspective de l'établissement d'un numéro unique pour la gestion sécuritaire des médicaments, l'Institute for Safe Medication Practices Canada et ses partenaires ont proposé d'apposer sur tous les formats d'emballage d'un médicament un code-barres 2D intégrant le format d'emballage, le numéro du fabricant, le numéro du produit et plus tard le lot et la péremption dans un format prévisible et lisible par différents types de lecteurs ${ }^{13}$. La mise en place de ce standard est urgente compte tenu que l'industrie recourt à des codes-barres, dont le contenu n'est pas forcément intelligible et pertinent pour une utilisation en établissement de santé à large échelle.

\section{CIRCUIT DU MÉDICAMENT}

Jodoin et coll. ont publié en 2005 un rapport sur les systèmes automatisés et robotisés de distribution des médicaments. ${ }^{7}$ Nous avons participé à cette initiative québécoise en établissement, notamment à la constitution du cadre de référence du circuit du médicament et de ses 54 étapes et d'une grille de critères de conformité. À partir du concept d'identité numérique, notre équipe de recherche a établi les possibilités d'intégration du contenu numérique (image et plus tard son) aux différentes étapes du circuit du médicament. En bref, l'image pourra être affichée de différentes façons : soit en consultation directe sur l'intranet (c.-à-d. par la consultation de 
la liste des médicaments et de l'affichage d'une ou de plusieurs images liées à un médicament, par l'affichage en temps réel des activités de service/renouvellement de service de médicaments ou l'administration de médicaments au chevet du patient) en production à la pharmacie (c.-à-d. par l'affichage de la quantité et de l'état de la production c'est-à-dire : à préparer, préparé, vérifié, contrôle de qualité), et à l'étage (c.-à-d. par l'affichage en temps réel sur un lecteur de codes-barres l'action en cours et la photo pertinente, notamment pour le remplissage de réserves d'étages ou de cabinets décentralisés). Selon l'étape et l'utilisateur concerné au cours de la chaîne du circuit du médicament dans l'hôpital, l'interface comportant l'identité numérique peut fournir des informations relatives au médicament, à sa quantité, la ou les photos pertinentes, l'identité du patient (numéro de dossier, épisode de soins, prénom - nom, chambre). Le tableau 1 reprend ces étapes et établit celles qui peuvent bénéficier d'une banque de données de photos et de sons permettant de confirmer l'identité numérique d'un médicament.

\section{DÉVELOPPEMENT D'UN LABORATOIRE PHOTO}

Le centre hospitalier universitaire (CHU) Sainte-Justine est un centre mère-enfant. Le département de pharmacie offre une distribution unitaire quotidienne ou hebdomadaire. Il effectue chaque année près d'un million de transactions informatiques, près de 400000 préparations magistrales stériles et non stériles et près de 18000 lignes de commandes auprès $\mathrm{du}$ distributeur et des fabricants.

À défaut de pouvoir utiliser une banque de données exhaustive d'images de médicaments disponibles à l'échelle canadienne, nous avons développé un laboratoire photo permettant, de façon efficace, reproductible et peu coûteuse, la prise de photos des médicaments utilisés au CHU SainteJustine dans le cadre du circuit du médicament. Avant de déterminer les caractéristiques du laboratoire, nous avons comparé les appareils photo numériques disponibles sur le marché et, sur les conseils d'un spécialiste en photo, nous avons retenu le Canon EOS Rebel XSi 12,2 mégapixels avec objectif EFS, 18-55 mm - 3,5-5,6 et déclencheur à distance. L'appareil photo pèse environ $500 \mathrm{~g}$, est doté d'un écran de 3 pouces et relié à un ordinateur par un câble USB d'interface IFC-200U. La prise des images est effectuée à l'aide du logiciel Canon EOS Utility. Compte tenu du transfert immédiat des images vers l'ordinateur au fur et à mesure des prises de vue de l'appareil photo, nous n'avons pas acheté de carte mémoire additionnelle. L'ensemble a coûté environ 1300 \$.

Afin de prendre des photos de la qualité désirée, nous avons recherché, par essais et erreurs, un environnement physique optimal offrant un a) rétro-éclairage adéquat (c.-à-d. les contours et inscriptions sur les formes posologiques photographiées sont lisibles et l'apparence des formes est comparable à l'examen visuel), b) une surface de contraste inclinée de $\mathrm{x}^{\circ}$ (donnant une meilleure perspective du produit lorsque cela est nécessaire), rainurée (afin d'éviter que le produit ne se déplace) et polyvalente, c) un système de portes battantes permettant l'introduction de produits de différentes dimensions, d) un support intégré pour fixer l'appareil photo, e) un matériau peu coûteux et une dimension optimale pour assurer une distance focale acceptable entre l'appareil photo et les médicaments à photographier. De plus, le laboratoire doit être déposé sur un bureau de travail pour que nous soyons en mesure de l'intégrer à nos activités de gestion des stocks. A priori, une distance focale de $29 \mathrm{~cm}$ du plan de travail a été retenue. La hauteur de la chambre photo a été influencée par la taille des produits les plus gros à photographier (p. ex. solutés de 3 ou $5 \mathrm{~L}$ ).

Après une semaine d'apprentissage par essais et erreurs avec une structure temporaire en carton, nous avons établi les dimensions définitives d'un boîtier, qui a été réalisé par notre équipe des services techniques. Il est en bois stratifié aggloméré d'une épaisseur de $2 \mathrm{~cm}$, d'une largeur de $64 \mathrm{~cm}$, d'une longueur de $81 \mathrm{~cm}$ et d'une hauteur de $79 \mathrm{~cm}$. La surface intérieure stratifiée est de couleur blanche. L'éclairage est constitué de deux tubes fluorescents situés à l'intérieur du laboratoire, à la base, en position latérale gauche et droite du plan de travail, protégés par des panneaux de plastiques blancs semi-transparents destinés à diffuser la lumière de façon homogène. Les tubes fluorescents peuvent être allumés de façon indépendante, ce qui permet une plus grande flexibilité d'éclairage. Une tige métallique est fixée verticalement à l'intérieur du laboratoire, derrière la jonction entre les deux battants de porte. Elle sert de point d'ancrage au pied flexible de l'appareil photo. Le pied flexible est fixé de façon perpendiculaire et peut glisser sur la tige métallique de haut en bas afin de faire varier la distance focale ou de permettre un pivot de gauche à droite. Le fond du boîtier est muni d'un panneau de mélamine texturé de couleur bleue, d'une règle transparente utilisée comme jalon, qui permet l'évaluation de la taille réelle des médicaments. Un poste informatique avec écran plat, clavier et souris a été intégré au laboratoire. L’ensemble du câblage permettant de relier le déclencheur à l'appareil photo et l'appareil photo au poste informatique est possible grâce à une ouverture à l'arrière du boîtier. Le boîtier peut être déposé et utilisé sur une surface de travail pour une personne en position assise (table de $30 \mathrm{~cm}$ de haut) ou debout (comptoir de $36 \mathrm{~cm}$ de haut). La figure 1 illustre le laboratoire de photo. Le tableau 2 présente des exemples de problèmes observés et les solutions apportées au cours du développement du concept.

\section{BANQUE DE DONNÉES}

Durant le développement du concept, nous avons choisi de créer une banque de données parallèle aux banques de 
Tableau 1. Étapes du circuit du médicament et identité numérique

\begin{tabular}{|c|c|c|c|c|}
\hline Étapes & Localisation & Principaux intervenants & $\begin{array}{l}\text { Description } \\
\text { dechaque étape }\end{array}$ & $\begin{array}{l}\text { Modalités d'utilisation } \\
\text { de la banque de photos }\end{array}$ \\
\hline 1 & Établissement & Comité de pharmacologie & $\begin{array}{l}\text { Évaluation pour ajout à la } \\
\text { liste locale }\end{array}$ & $\begin{array}{l}\text { Favorise les produits avec identité } \\
\text { numérique complète }\end{array}$ \\
\hline$\overline{2}$ & Pharmacie & $\begin{array}{l}\text { Chef du département } \\
\text { de pharmacie }\end{array}$ & $\begin{array}{l}\text { Décision d'ajout ou de } \\
\text { modification à la liste locale } \\
\text { et règle }\end{array}$ & $\begin{array}{l}\text { Favorise les produits avec identité numérique } \\
\text { complète }\end{array}$ \\
\hline$\overline{3}$ & Établissement & $\begin{array}{l}\text { Exécutif du conseil des } \\
\text { médecins, dentistes et } \\
\text { pharmaciens }\end{array}$ & $\begin{array}{l}\text { Approbation de la décision } \\
\text { d'ajout ou de modification }\end{array}$ & $\begin{array}{l}\text { Facilite la diffusion d'une information } \\
\text { complète après l'ajout d'un médicament }\end{array}$ \\
\hline$\overline{4}$ & Groupe d'achats & Comité des pharmaciens & $\begin{array}{l}\text { Mandat, appel d'offres et } \\
\text { ententes }\end{array}$ & $\begin{array}{l}\text { Utilisation des images (étiquettes et produits) } \\
\text { lors de l'évaluation pour octroi de contrats }\end{array}$ \\
\hline 5 & Pharmacie & $\begin{array}{l}\text { Technicien, assistant- } \\
\text { technique, pharmacien }\end{array}$ & $\begin{array}{l}\text { Création du produit dans } \\
\text { les différents systèmes }\end{array}$ & $\begin{array}{l}\text { Ajout des images et autres données } \\
\text { pertinentes à la banque de données locales }\end{array}$ \\
\hline 6 & Pharmacie & $\begin{array}{l}\text { Technicien, assistant- } \\
\text { technique }\end{array}$ & $\begin{array}{l}\text { Achat du médicament par } \\
\text { le grossiste ou fabricant }\end{array}$ & $\begin{array}{l}\text { Confirmation de la lecture du code-barres } \\
\text { avec le son et l'image lors de commandes }\end{array}$ \\
\hline 7 & Pharmacie & $\begin{array}{l}\text { Technicien, assistant- } \\
\text { technique }\end{array}$ & $\begin{array}{l}\text { Réception du médicament } \\
\text { et traçabilité }\end{array}$ & $\begin{array}{l}\text { Affichage de l'image sur écran lors de la } \\
\text { réception et de l'identification des produits } \\
\text { dont l'identité numérique a changé }\end{array}$ \\
\hline 8 & Pharmacie & $\begin{array}{l}\text { Technicien, assistant- } \\
\text { technique }\end{array}$ & $\begin{array}{l}\text { Gestion des drogues } \\
\text { contrôlées et stupéfiants } \\
\text { selon la « loi sur les autres } \\
\text { substances » }\end{array}$ & $\begin{array}{l}\text { Les données de réfractométrie permettent } \\
\text { d'évaluer la pureté des médicaments } \\
\text { retournés }\end{array}$ \\
\hline$\overline{9}$ & Pharmacie & $\begin{array}{l}\text { Technicien, assistant- } \\
\text { technique }\end{array}$ & Entreposage & $\begin{array}{l}\text { Confirmation de l'emplacement avec le } \\
\text { code-barres ou les données radio- } \\
\text { identification }\end{array}$ \\
\hline 10 & Pharmacie & $\begin{array}{l}\text { Technicien, assistant- } \\
\text { technique }\end{array}$ & $\begin{array}{l}\text { Saisie informatique de la } \\
\text { réception }\end{array}$ & $\begin{array}{l}\text { Affichage de l'image sur écran lors de la } \\
\text { réception OU prise de photo et saisie des } \\
\text { informations complémentaires si le produit } \\
\text { est inconnu, mise à jour de la banque de } \\
\text { données }\end{array}$ \\
\hline$\overline{11}$ & Pharmacie & $\begin{array}{l}\text { Technicien, assistant- } \\
\text { technique }\end{array}$ & $\begin{array}{l}\text { Emballage en lots, lorsque } \\
\text { requis }\end{array}$ & $\begin{array}{l}\text { Affichage de l'image sur écran lors de } \\
\text { l'emballage, confirmation du produit avec } \\
\text { le code-barres }\end{array}$ \\
\hline 12 & Pharmacie & $\begin{array}{l}\text { Technicien, assistant- } \\
\text { technique }\end{array}$ & $\begin{array}{l}\text { Préparation en lots, lorsque } \\
\text { requis (stériles, non stériles) }\end{array}$ & $\begin{array}{l}\text { Affichage de l'image sur écran lors de la } \\
\text { préparation, confirmation du produit avec } \\
\text { le code-barres }\end{array}$ \\
\hline$\overline{13}$ & Pharmacie & $\begin{array}{l}\text { Technicien, assistant- } \\
\text { technique }\end{array}$ & $\begin{array}{l}\text { Étiquetage si emballage ou } \\
\text { préparation (avec ou sans } \\
\text { code-barres) }\end{array}$ & $\begin{array}{l}\text { Lecture du code-barres pour faire le lien } \\
\text { entre le service et le produit }\end{array}$ \\
\hline$\overline{14}$ & Unités/cliniques & $\begin{array}{l}\text { Médecin ou autre } \\
\text { professionnel }\end{array}$ & $\begin{array}{l}\text { Rédaction d'une } \\
\text { ordonnance }\end{array}$ & $\begin{array}{l}\text { Affichage de l'image et d'autres informations } \\
\text { sur les différentes présentations du produit } \\
\text { sur écran lors de prescriptions }\end{array}$ \\
\hline$\overline{15}$ & Unités/cliniques & $\begin{array}{l}\text { Infirmière, infirmière } \\
\text { auxiliaire }\end{array}$ & Relevé de l'ordonnance & $\begin{array}{l}\text { Des codes-barres sur les ordonnances } \\
\text { préimprimées permettent d'identifier avec } \\
\text { certitude les médicaments au commun }\end{array}$ \\
\hline 16 & Unités/cliniques & $\begin{array}{l}\text { Infirmière, infirmière } \\
\text { auxiliaire, commis, messager }\end{array}$ & $\begin{array}{l}\text { Envoi de l'ordonnance à la } \\
\text { pharmacie }\end{array}$ & \\
\hline 17 & Pharmacie & $\begin{array}{l}\text { Technicien, assistant- } \\
\text { technique }\end{array}$ & $\begin{array}{l}\text { Réception de l'ordonnance } \\
\text { et priorisation }\end{array}$ & \\
\hline 18 & Pharmacie & $\begin{array}{l}\text { Technicien, assistant- } \\
\text { technique }\end{array}$ & $\begin{array}{l}\text { Saisie informatique au } \\
\text { dossier pharmacologique }\end{array}$ & $\begin{array}{l}\text { Affichage de l'image sur écran lors de la } \\
\text { saisie }\end{array}$ \\
\hline$\overline{19}$ & Pharmacie & Pharmacien & $\begin{array}{l}\text { Validation (âge, poids, taille, } \\
\text { duplication, allergie, } \\
\text { intolérance, duplication, } \\
\text { interaction, pertinence, } \\
\text { dose etc.) }\end{array}$ & $\begin{array}{l}\text { Affichage de l'image sur écran lors de } \\
\text { la validation }\end{array}$ \\
\hline 20 & Pharmacie & Pharmacien & Intervention, lorsque requis & $\begin{array}{l}\text { Au besoin, intégration de l'image à la note } \\
\text { d'intervention }\end{array}$ \\
\hline 21 & Pharmacie & Pharmacien & Envoi de l'intervention & \\
\hline
\end{tabular}


Tableau 1. Étapes du circuit du médicament et identité numérique (suite)

\begin{tabular}{|c|c|c|c|c|}
\hline Étapes & Localisation & Principaux intervenants & $\begin{array}{c}\text { Description } \\
\text { dechaque étape }\end{array}$ & $\begin{array}{c}\text { Modalités d'utilisation } \\
\text { de la banque de photos }\end{array}$ \\
\hline 22 & Pharmacie & Pharmacien & $\begin{array}{l}\text { Gestion des exceptions } \\
\text { (hors-liste, programme } \\
\text { d'accès spécial, recherche } \\
\text { clinique) }\end{array}$ & $\begin{array}{l}\text { Identification grâce à l'identité numérique } \\
\text { ou à l'absence de celle-ci; affichage de } \\
\text { l'image sur écran lors de saisie }\end{array}$ \\
\hline$\overline{23}$ & Pharmacie & $\begin{array}{l}\text { Technicien, assistant- } \\
\text { technique }\end{array}$ & $\begin{array}{l}\text { Préparation individuelle, } \\
\text { lorsque requis }\end{array}$ & $\begin{array}{l}\text { Affichage de l'image sur écran lors de } \\
\text { préparation, confirmation du produit avec } \\
\text { le code-barres }\end{array}$ \\
\hline 24 & Pharmacie & $\begin{array}{l}\text { Technicien, assistant- } \\
\text { technique }\end{array}$ & $\begin{array}{l}\text { Emballage individuel, } \\
\text { lorsque requis }\end{array}$ & $\begin{array}{l}\text { Affichage de l'image sur écran lors de } \\
\text { préparation, confirmation du produit avec } \\
\text { le code-barres }\end{array}$ \\
\hline 25 & Pharmacie & $\begin{array}{l}\text { Technicien, assistant- } \\
\text { technique }\end{array}$ & Étiquetage, lorsque requis & $\begin{array}{l}\text { Affichage de l'image sur écran lors de } \\
\text { préparation, confirmation du produit avec } \\
\text { le code-barres }\end{array}$ \\
\hline 26 & Pharmacie & $\begin{array}{l}\text { Pharmacien, assistant- } \\
\text { technique }\end{array}$ & $\begin{array}{l}\text { Vérification contenant- } \\
\text { contenu }\end{array}$ & $\begin{array}{l}\text { Affichage de l'image sur écran lors de } \\
\text { préparation, confirmation du produit avec } \\
\text { le code-barres }\end{array}$ \\
\hline 27 & Pharmacie & $\begin{array}{l}\text { Messager, assistant- } \\
\text { technique, commis }\end{array}$ & $\begin{array}{l}\text { Expédition des premières } \\
\text { doses de médicaments }\end{array}$ & $\begin{array}{l}\text { Affichage de l'image sur écran lors de } \\
\text { préparation, confirmation du produit avec } \\
\text { le code-barres }\end{array}$ \\
\hline 28 & Pharmacie & $\begin{array}{l}\text { Messager, assistant- } \\
\text { technique, commis }\end{array}$ & $\begin{array}{l}\text { Renouvellement de service } \\
\text { quotidien ou à fréquence } \\
\text { variable des médicaments }\end{array}$ & $\begin{array}{l}\text { Affichage de l'image sur écran lors de } \\
\text { préparation, confirmation du produit avec } \\
\text { le code-barres }\end{array}$ \\
\hline 29 & Pharmacie & $\begin{array}{l}\text { Messager, assistant- } \\
\text { technique, commis }\end{array}$ & $\begin{array}{l}\text { Emballage et préparation } \\
\text { des congés temporaires de } \\
\text { patients }\end{array}$ & $\begin{array}{l}\text { Affichage de l'image sur écran lors de } \\
\text { préparation, confirmation du produit avec } \\
\text { le code-barres }\end{array}$ \\
\hline 30 & Unités/cliniques & $\begin{array}{l}\text { Commis-intermédiaire, } \\
\text { préposé, infirmière }\end{array}$ & Réception & \\
\hline 31 & Unités/cliniques & $\begin{array}{l}\text { Commis-intermédiaire, } \\
\text { préposé, infirmière }\end{array}$ & Entreposage & $\begin{array}{l}\text { Confirmation de l'emplacement avec le } \\
\text { code-barres ou les données de radio- } \\
\text { identification }\end{array}$ \\
\hline$\overline{32}$ & Unités/cliniques & $\begin{array}{l}\text { Infirmière, infirmière } \\
\text { auxiliaire }\end{array}$ & $\begin{array}{l}\text { Planification des doses à } \\
\text { administrer }\end{array}$ & $\begin{array}{l}\text { Affichage de l'image sur écran lors de } \\
\text { préparation, confirmation du produit avec } \\
\text { le code-barres }\end{array}$ \\
\hline 33 & Unités/cliniques & $\begin{array}{l}\text { Infirmière, infirmière } \\
\text { auxiliaire }\end{array}$ & Préparation, lorsque requis & $\begin{array}{l}\text { Affichage de l'image sur écran lors de la } \\
\text { préparation, confirmation du produit avec } \\
\text { le code-barres }\end{array}$ \\
\hline 34 & Unités/cliniques & $\begin{array}{l}\text { Infirmière, infirmière } \\
\text { auxiliaire }\end{array}$ & Étiquetage, lorsque requis & $\begin{array}{l}\text { Étiquetage généré à partir des données } \\
\text { de l'identité numérique }\end{array}$ \\
\hline 35 & Unités/cliniques & $\begin{array}{l}\text { Infirmière, infirmière } \\
\text { auxiliaire }\end{array}$ & $\begin{array}{l}\text { Tournée des chambres et } \\
\text { administration du } \\
\text { médicament au patient }\end{array}$ & $\begin{array}{l}\text { Affichage de l'image sur écran lors de la } \\
\text { préparation, confirmation du produit avec } \\
\text { le code-barres }\end{array}$ \\
\hline 36 & Unités/cliniques & $\begin{array}{l}\text { Infirmière, infirmière } \\
\text { auxiliaire }\end{array}$ & $\begin{array}{l}\text { Vérification de l'identité, du } \\
\text { médicament, de l'horaire } \\
\text { et de la dose à administrer }\end{array}$ & $\begin{array}{l}\text { Affichage de l'image sur écran lors de la } \\
\text { préparation, confirmation du produit avec } \\
\text { le code-barres, prononciation du nom du } \\
\text { médicament }\end{array}$ \\
\hline 37 & Unités/cliniques & $\begin{array}{l}\text { Infirmière, infirmière } \\
\text { auxiliaire }\end{array}$ & $\begin{array}{l}\text { Enregistrement les doses } \\
\text { administrées }\end{array}$ & $\begin{array}{l}\text { Affichage de l'image sur écran lors de la } \\
\text { préparation, confirmation du produit avec } \\
\text { le code-barres }\end{array}$ \\
\hline 38 & Unités/cliniques & $\begin{array}{l}\text { Médecin, pharmacien et } \\
\text { autre professionnel }\end{array}$ & $\begin{array}{l}\text { Évaluation de la } \\
\text { phamacothérapie du patient }\end{array}$ & \\
\hline 39 & Unités/cliniques & $\begin{array}{l}\text { Infirmière, infirmière } \\
\text { auxiliaire }\end{array}$ & $\begin{array}{l}\text { Gestion des déchets de } \\
\text { médicaments }\end{array}$ & \\
\hline$\overline{40}$ & Unités/cliniques & $\begin{array}{l}\text { Infirmière, infirmière } \\
\text { auxiliaire }\end{array}$ & $\begin{array}{l}\text { Gestion de la documentation } \\
\text { y compris les stupéfiants et } \\
\text { drogues contrôlées }\end{array}$ & \\
\hline$\overline{41}$ & Unités/cliniques & $\begin{array}{l}\text { Assistant-technique, } \\
\text { pharmacien }\end{array}$ & $\begin{array}{l}\text { Impression des rapports pour } \\
\text { les infirmières, médecins }\end{array}$ & $\begin{array}{l}\text { Ajout de l'image et des codes-barres } \\
\text { lorsque requis sur certains rapports }\end{array}$ \\
\hline$\overline{42}$ & Unités/cliniques & $\begin{array}{l}\text { Infirmière, infirmière } \\
\text { auxiliaire, médecins, autres } \\
\text { professionnels }\end{array}$ & $\begin{array}{l}\text { Communications avec la } \\
\text { pharmacie (technique ou } \\
\text { pharmaceutique) }\end{array}$ & \\
\hline
\end{tabular}


Tableau 1. Étapes du circuit du médicament et identité numérique (suite et fin)

\begin{tabular}{|c|c|c|c|c|}
\hline Étapes & Localisation & Principaux intervenants & $\begin{array}{l}\text { Description } \\
\text { dechaque étape }\end{array}$ & $\begin{array}{l}\text { Modalités d'utilisation } \\
\text { de la banque de photos }\end{array}$ \\
\hline$\overline{43}$ & Unités/cliniques & Pharmacien & $\begin{array}{l}\text { Enseignement aux étudiants } \\
\text { en pharmacie, aux } \\
\text { professionnels de } \\
\text { l'établissement }\end{array}$ & \\
\hline$\overline{44}$ & Unités/cliniques & Assistant-technique & $\begin{array}{l}\text { Remplissage de l'armoire } \\
\text { de nuit }\end{array}$ & $\begin{array}{l}\text { Affichage de l'image sur écran lors de } \\
\text { la préparation, confirmation du produit } \\
\text { avec le code-barres }\end{array}$ \\
\hline$\overline{45}$ & Unités/cliniques & Assistant-technique & $\begin{array}{l}\text { Remplissage des plateaux } \\
\text { d'urgence, des chariots de } \\
\text { réanimation }\end{array}$ & $\begin{array}{l}\text { Affichage de l'image sur écran lors de } \\
\text { la préparation, confirmation du produit } \\
\text { avec le code-barres }\end{array}$ \\
\hline 46 & Unités/cliniques & Assistant-technique & $\begin{array}{l}\text { Remplissage des réserves } \\
\text { d'étage (médicaments au } \\
\text { commun) }\end{array}$ & $\begin{array}{l}\text { Affichage de l'image sur écran lors de } \\
\text { préparation, confirmation du produit } \\
\text { avec le code-barres }\end{array}$ \\
\hline 47 & Pharmacie & Pharmacien & $\begin{array}{l}\text { Soins pharmaceutiques } \\
\text { à l'étage }\end{array}$ & Élaboration de fiches-conseils avec images \\
\hline 48 & Pharmacie & Pharmacien & $\begin{array}{l}\text { Participation aux activités } \\
\text { paracliniques } \\
\text { interdisciplinaires }\end{array}$ & \\
\hline 49 & Pharmacie & Assistant-technique & $\begin{array}{l}\text { Gestion des retours pour } \\
\text { récupération }\end{array}$ & $\begin{array}{l}\text { Affichage de l'image sur écran lors de la } \\
\text { préparation, confirmation du produit } \\
\text { avec le code-barres }\end{array}$ \\
\hline 50 & Pharmacie & $\begin{array}{l}\text { Technicien, assistant-technique, } \\
\text { pharmacien }\end{array}$ & $\begin{array}{l}\text { Gestion de la documentation } \\
\text { et des archives selon } \\
\text { calendrier de conservation }\end{array}$ & $\begin{array}{l}\text { Archivage permanent des stocks utilisés } \\
\text { dans les différentes étapes du circuit du } \\
\text { médicament }\end{array}$ \\
\hline 51 & Pharmacie & Technicien, assistant-technique & $\begin{array}{l}\text { Gestion des produits } \\
\text { périmés pour crédits au } \\
\text { fabricant/grossiste }\end{array}$ & \\
\hline 52 & Établissement & Comité de pharmacologie & Réévaluation de I'utilisation & \\
\hline 53 & Établissement & $\begin{array}{l}\text { Technicien, assistant-technique, } \\
\text { pharmacien }\end{array}$ & $\begin{array}{l}\text { Déclaration et divulgation } \\
\text { des accidents/incidents }\end{array}$ & Archivage des preuves et comparaison \\
\hline$\overline{54}$ & Groupe d'achats & Comité des pharmaciens & Plaintes, ruptures, & $\begin{array}{l}\text { Meilleure description des plaintes } \\
\text { étiquetages, etc. }\end{array}$ \\
\hline
\end{tabular}

\section{Tableau 2. Problèmes observés et solutions apportées lors du développement du concept}

Problème

Taille variable des formes posologiques (p. ex. comprimés c. solutés de $3 \mathrm{~L}$ )

Renseignements parfois illisibles à la surface des

formes posologiques

Renseignements parfois difficiles à capter en un seul cliché compte tenu de la forme ronde de l'objet

Difficulté d'immobiliser certains formats

Difficulté de photographier des liquides transparents
Solution apportée

Utilisation d'une lentille appropriée comportant le mode macro avec un laboratoire de taille suffisante

Ajustement de l'éclairage avec rétro-éclairage et plancher foncé

Positionnement adéquat du médicament avec mise au point

sur les renseignements les plus pertinents

Utilisation d'un plan incliné rainuré

Utilisation d'une cupule posée sur fond mat données existantes pour la gestion des stocks (GRM-Espresso, Logibec, QC) et pour le dossier pharmacologique informatisé (GesPharx, CGSI Solutions TI Inc) afin de permettre des modifications et des ajouts en cours de route. Toutefois, nous pensons que cette banque de données devrait être finalement intégrée aux applications commerciales, si les fabricants acceptent de collaborer afin d'assurer une intégrité référentielle.

À l'aide du logiciel SQL Server, nous avons développé une banque de données comportant trois tables. La table [Médicaments] contient les variables suivantes : numéro unique de médicament, nom générique, voie d'administration, forme, teneur, unité de teneur, unité de volume associée à la teneur si cela est applicable, commentaires sur d'autres caractéristiques du médicament. La table [Produits] contient les variables suivantes : numéro unique de produit, nom commercial, code-barres si cela est applicable, type d'emballage du codebarres (c.-à-d. caisse, bouteille, dose), forme, format, unité de format, commentaires, DIN, fabricant, commentaires sur d'autres caractéristiques du produit et numéro unique du médicament. Enfin, la table [Données numériques] contient les 


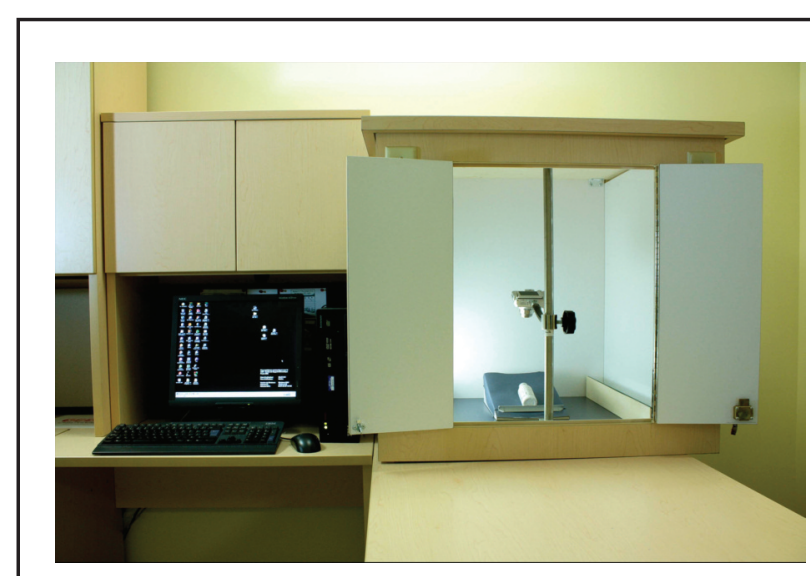

Figure 1. Laboratoire photo pour médicaments en établissement de santé.

variables suivantes : nom de la photo, numéro unique du produit. Cette table pourra contenir tout autre document numérique (p. ex. documents en .pdf, enregistrement sonore du nom commercial et générique en .wav)

En ce qui concerne les photos, les images obtenues sont sauvegardées en format tif et sont nommées d'après le numéro unique attribué lors de la création du produit. Les images sont enregistrées en format .jpg pour pouvoir être publiées dans la banque à la résolution maximale de l'appareil photo, soit 12 mégapixels. Les images seront ensuite redimensionnées en lots afin de correspondre aux nécessités des logiciels.

La figure 2 illustre deux exemples de fiches produites par la banque de données avec les photos qui leur sont associées.

\section{PHASE PILOTE}

Une fois la structure de la base de données définie et le laboratoire photo éprouvé, nous avons lancé une phase pilote. Nous nous sommes concentrés sur les produits oraux liquides et les formes sèches sous forme de comprimés disponibles sur le marché. Pour chaque produit disponible en stock et inscrit à la liste de médicaments du CHU Sainte-Justine, nous avons convenu de prendre au moins deux photos, soit le contenant original (bouteille, flacon), le contenu (comprimé, poudre non diluée ou liquide) et, si cela était applicable, le contenu dans son format final d'utilisation (poudre diluée). Afin de présenter le contenu des formes liquides, nous avons retenu des nacelles de plastique de $4,5 \times 4,5 \times 1 \mathrm{~cm}$ et une quantité d'environ $3 \mathrm{~mL}$

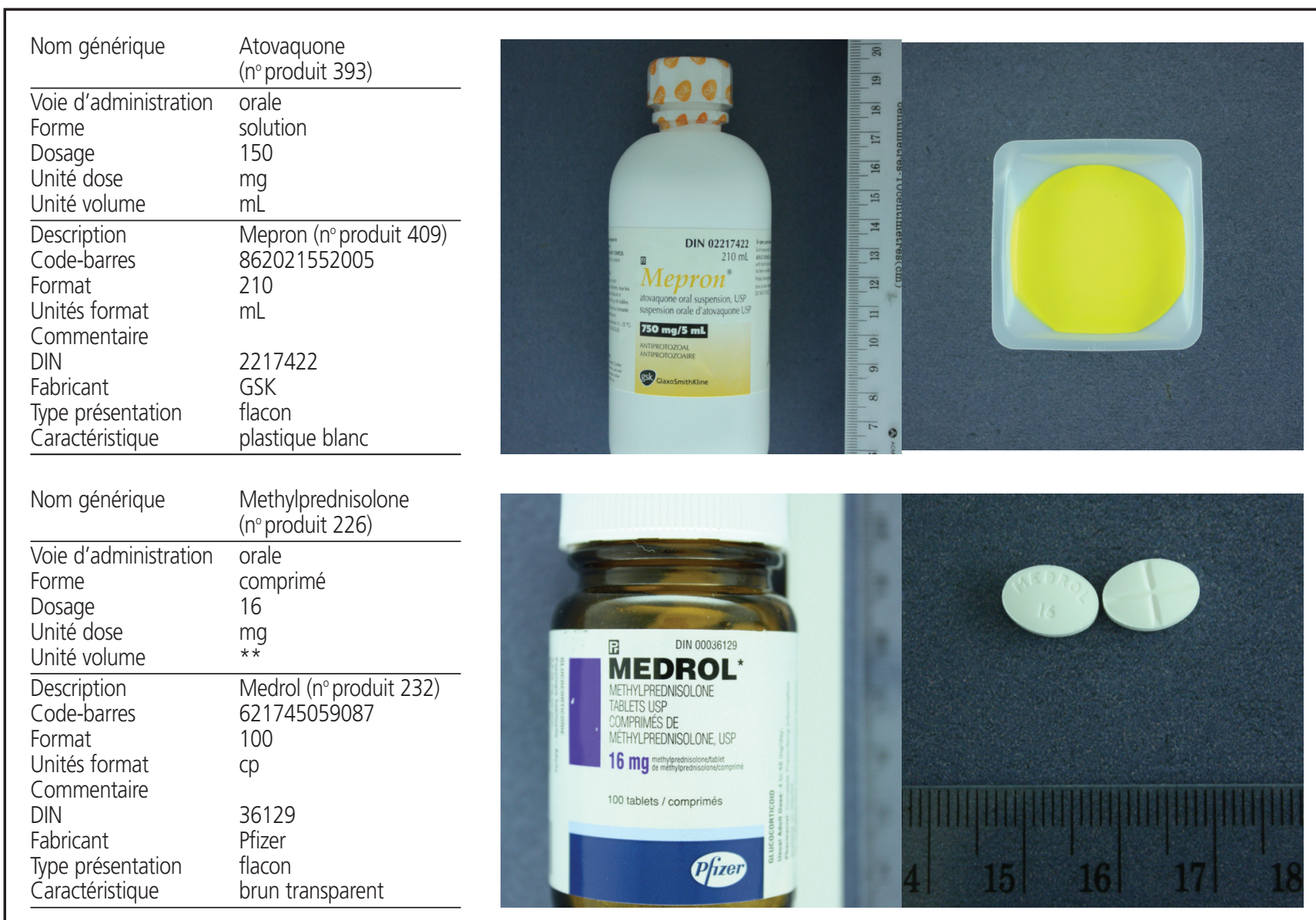

Figure 2. Deux exemples de fiches produits de la banque de données avec photos associées. 
à y déposer pour la prise de la photo. Après les tests effectués, nous avons constaté que les images sont de meilleure qualité en utilisant qu'un seul des deux néons et en annulant le filtre de lumière proposé par le logiciel d'édition de photos. Chaque produit ou sous-produit est placé sur le plan incliné, les portes sont fermées, et le cliché est obtenu par déclencheur à distance. Chaque fichier obtenu est renommé selon la nomenclature, sauvegardé sur le serveur pharmacie et intégré à la banque de données, la saisie des données pertinentes du produit comprise.

Le projet pilote s'est déroulé de juin à décembre 2008, y compris le développement du concept (3 mois), la fabrication du laboratoire de photos (2 semaines), le développement de la banque de données (8 heures), la création de fiches produits avec prise de photos (environ 15 minutes/produit, c.-à-d. 40 heures pour 138 produits et 277 images).

\section{PROCHAINES ÉTAPES ET PERSPECTIVES}

Le concept démontre qu'il est possible de mettre en place un laboratoire photo dans un département de pharmacie pour l'intégration de la gestion de l'identité numérique d'un produit dans le cadre du circuit du médicament. À partir de cette phase pilote, nous avons entrepris le développement de la gestion de la production des préparations magistrales non stériles à partir de la banque de données des images. Ainsi, au terme du développement, le personnel technique et pharmaceutique sera informé, à l'écran et en temps réel, des doses orales liquides à préparer en ayant l'ensemble des données requises pour la préparation, c.-à-d. nom du patient avec double identifiant, localisation du patient, le médicament à préparer, le produit source y compris les photos du contenant et du contenu, la quantité à préparer et le positionnement du piston. Cette mise en application du concept fera l'objet d'une évaluation distincte.

Dans le futur, nous souhaitons que cette banque de données soit exploitée jusqu'aux étages par le personnel de la pharmacie ou les autres soignants. Par exemple, une erreur d'ensachage d'un médicament est difficilement décelable si l'infirmière ne peut contre-vérifier l'apparence du comprimé qu'elle obtient sans déchirer le sachet, alors qu'un doute subsiste dans son esprit. De même, le pharmacien peut difficilement vérifier une seringue orale préparée par un assistant-technique s'il ne peut comparer l'apparence de la solution préparée à la solution originale contenue dans le contenant du fabricant. Enfin, l'émergence de contrefaçons de médicaments à l'échelle internationale ajoute à la nécessité de mise en place d'un outil d'identification permettant de vérifier, tout au long du circuit du médicament, l'identité du produit utilisé.

\section{CONDITIONS DE SUCCÈS}

L'intégration de photos et de sons liés aux médicaments est un outil qui peut être utile à la prestation sécuritaire de médicaments. Cette intégration sera réalisée à large échelle, notamment si un standard unique d'identification par codesbarres, qui comporte un numéro unique propre à chaque type d'emballage, émerge au Canada, si les sociétés pharmaceutiques sont tenues de fournir, dans un format structuré et standardisé, les images de contenants et de contenus et si une banque de données canadienne est accessible au réseau de la santé à peu de frais. À notre avis, ces données font partie de l'identité du médicament au même titre que la monographie et devraient être disponibles à tous.

\section{Références}

1. Laccès aux produits thérapeutiques. Le processus de réglementation au Canada. Ottawa (ON) : Santé Canada. Publié à http:// www.hc-sc.gc.ca/ahc-asc/pubs/hpfb-dgpsa/access-therapeutic_ acces-therapeutique-fra.php. Consulté le 4 février 2009.

2. Médicaments et produits de santé. Questions des détaillants. Ottawa (ON) : Santé Canada. Publié à http://www.hc-sc.gc.ca/dhp-mps/ prodnatur/faq/question_retailers-detaillants-fra.php. Consulté le 4 février 2009.

3. Bussières JF, Thibault M, Lebel D, Prot-Labarthe S. Conformity of commercial oral single solid unit dose package in hospital pharmacy practice. Int J Qual Health 2008;20(3);206-210.

4. American Society of Hospital Pharmacists. ASHP statement on unit dose drug distribution. Am J Hosp Pharm 1989;46:2346. Publié à http:// www.ashp.org/DocLibrary/BestPractices/UnitDose.aspx. Consulté le 4 février 2009.

5. Canadian Society of Hospital Pharmacists 2015 - a practice excellence initiative for pharmacists in hospitals and related healthcare settings. Publié à http://www.cshp.ca/programs/cshp2015/index_e.asp. Consulté le 18 août 2009.

6. Babich M, Bussières JF, Hall KW, Harding J, Johnson N, Lefebvre P, et al., rédacteurs. Rapport annuel 2007-2008. Pharmacies hospitalières canadiennes. Comité de rédaction; 2008. Publié à http://www. lillyhospitalsurvey.ca/hpc2/content/rep_2008_tocF.asp. Consulté le 4 février 2009.

7. Jodoin J, Lantin S, Bussières JF, Bouchard S, Cohen E, Lussier-Labelle F, et al. Les systèmes automatisés et robotisés utilisés pour la distribution des médicaments dans les établissements de santé au Québec. Rapport et recommandations du groupe de travail. Publication $\mathrm{n}^{\circ}$ 05-719-01F. Québec (QC) : Ministère de la santé et des services sociaux; avril 2005 [diffusé en octobre 2005]. ISBN 2-550-45497-9.

8. Conroy S, Sweis D, Planner C, Yeung V, Collier J, Haines L, et al. Interventions to reduce dosing errors in children: a systematic review of the literature. Drug Saf 2007;30(12):1111-1125.

9. What are the "ten key elements" of the medication-use system? Dans : Frequently asked questions (FAQ). Horsham (PA) : Institute for Safe Medication Practices. Publié à www.ismp.org/faq.asp\#Question_3. Consulté le 18 août 2009.

10. Kutsuma N, Yamaura K, Hosaka S, Kasuga K, Koresawa T, Nagamura M, et al. [Evaluation of the efficacy of a medicine bag printed with a photograph of the medicine for the prevention of drug-dispensing errors]. Yakugaku Zasshi 2007;127(9):1515-1521. Japonais.

11. Fox LM, Pham KH, Dollar M. Using interactive digital images of products to teach pharmaceutics. Am J Pharm Educ 2007;71(3):1-8.

12. Bussières JF, Ericksson L, Lebel D, Touzin K, Brossard P, Brophy J. Évaluation de la capacité à extraire des données provenant des progiciels en pharmacie d'établissement (RQRUM). Pharmactuel 2008;41(2): $80-86$. 
13. Pharmaceutical bar coding to improve patient safety: options for technical standards in the Canadian environment. Proceedings of a stakeholder invitational roundtable. Toronto $(\mathrm{ON})$ : Institute for Safe Medication Practices Canada; 2008. Publié à http://www.ismp-canada.org/ download/BarCoding_Roundtable_Proceedings.pdf. Consulté le 18 août 2009.

Jean-François Bussières, , B.Pharm, M.Sc, M.B.A., F.C.S.H.P., travail à la Pharmacie, CHU Sainte-Justine, Montréal, Québec.

Denis Lebel, B. Pharm., M.Sc., F.C.S.H.P., travail à la Pharmacie, CHU Sainte-Justine, Montréal, Québec.

Serge Voytenko est interne en pharmacie, Université de Marseille, Marseille, France.

Guillaume Vaquer est interne en pharmacie, Université de Montpellier, Montpellier, France.
Addresse de correspondance :

Jean-François Bussières

Pharmacie

CHU Sainte-Justine

3175, chemin de la côte Sainte Catherine

Montréal (QC) H3T 1C5

courriel : jf.bussieres@ssss.gouv.qc.ca

\section{Remerciements}

Nous tenons à remercier les membres de l'équipe des services techniques du CHU Sainte-Justine. Nous adressons en particulier tous nos remerciements à Karine Touzin, assistante de recherche et coordonnatrice de l'Unité de recherche en pratique pharmaceutique. Cette preuve de concept a été présentée par affiche au Professional Practice Conference, qui s'est tenu du $1^{\mathrm{er}}$ au 3 février 2009 à Toronto, en Ontario.

\section{Exclusive Offer for CSHP Members!}

Get $5 \%$ off of the standard price of $\$ 90$ when you purchase at www.pharmacists.ca/promotions and enter promo code cshp0808. Order your copy today.

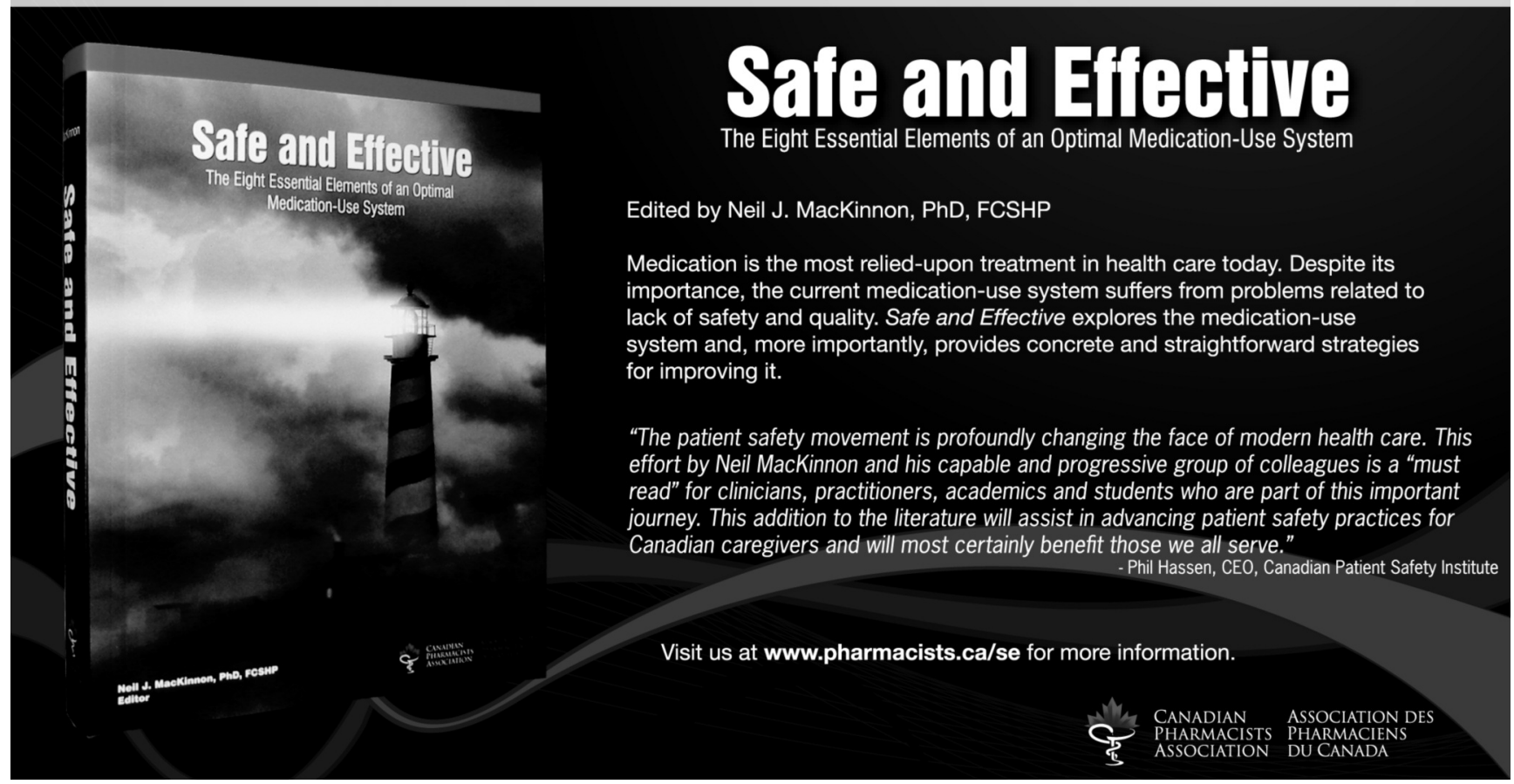

